\title{
Clinical efficacy of neoadjuvant chemsotherapy regimens FLEEOX vs. XELOX in patients with initially unresectable advanced gastric cancer: a propensity score analysis
}

\author{
Yang Li ${ }^{1,2, *}$, Jun Chen',*, Qi He ${ }^{2}$, Xiang $\mathrm{Ji}^{2}$, Xulin Wang ${ }^{2}$, Chaogang Fan² and Guoli Li \\ ${ }^{1}$ Division of Digestive Surgery, Xijing Hospital, Fourth Military Medical University, 710032, Xi'an, Shaanxi, China \\ ${ }^{2}$ Research Institute of General Surgery, Jinling Hospital, School of Medicine, Nanjing University, Nanjing 210002, China \\ *These authors have contributed equally to this work \\ Correspondence to: Guoli Li, email: liguoli82@126.com \\ Keywords: initially unresectable advanced gastric cancer, neoadjuvant chemotherapy, tumor response rate, overall survival, \\ chemotherapy-related toxicity \\ Received: February 23, $2017 \quad$ Accepted: May 15, $2017 \quad$ Published: June 28, 2017 \\ Copyright: Li et al. This is an open-access article distributed under the terms of the Creative Commons Attribution License 3.0 \\ (CC BY 3.0), which permits unrestricted use, distribution, and reproduction in any medium, provided the original author and source \\ are credited.
}

\section{ABSTRACT}

Purpose: This study was designed to assess the effectiveness of FLEEOX (5-Fu, leucovorin, etoposide, oxaliplatin, and epirubicin) compared with XELOX (capecitabine and oxaliplatin) as neoadjuvant chemotherapy (NAC) for initially unresectable advanced gastric cancer (AGC).

Methods: This study reviewed patients who underwent FLEEOX or XELOX for initially unresectable AGC. To reduce the bias in patient selection, we conducted propensity score match (PSM) with 1:1 ratio. Tumor and pathological response, surgical characteristics, chemotherapy-related toxicity and overall survival (OS) were analyzed.

Results: From January 2004 to December 2012, 436 patients were enrolled; 99 pairs of patients were generated after PSM. The tumor response rates were $80.8 \%$ and $68.7 \%$ in FLEEOX and XELOX $(P=0.018) .80$ patients $(80.8 \%)$ in FLEEOX and $63(63.6 \%)$ in $X E L O X$ received radical resection $(P<0.001)$. The pathological complete response rate and RO rate were $11.1 \%$ and $69.7 \%$ in FLEEOX, respectively, while $4.8 \%$ and $38.4 \%$ in XELOX $(P<0.001)$. Median OS time was longer in FLEEOX (30.0 vs. 25.1 months, $P<0.001)$. In addition, more toxicities occurred in FLEEOX, including leukocytopenia $(17.2 \%$ vs. $7.1 \%, P=0.024)$, nausea $(17.2 \%$ vs. $6.1 \%, P=0.012)$ and vomiting $(22.2 \%$ vs. $10.1 \%$, $P=0.016)$. The overall toxicity rate was higher in FLEEOX $(71.7 \%$ vs. $35.4 \%, P<0.001)$.

Conclusion: The FLEEOX regimen as NAC for patients with initially unresectable AGC can improve tumor response rate, radical resection rate, $\mathrm{RO}$ rate, and $\mathrm{OS}$ as compared to XELOX regimen. More chemotherapy-related toxicity was observed in FLEEOX group, although no chemotherapy-related deaths and aborting were observed. Further randomized clinical trials on the FLEEOX regimen are necessary.

\section{INTRODUCTION}

Gastric cancer is one of the leading cause of cancerrelated deaths worldwide [1] and causes 697,000 new diagnosed cases and 499,000 related deaths in China each year [2]. Moreover, $80 \%-90 \%$ gastric cancer patients in China are diagnosed at an advanced stage with extensive regional lymph node involvement or invasion of adjacent structures in first medical consultation [3, 4]. Additionally, only $50-60 \%$ of gastric cancer patients are suitable candidates for curative surgery [5]. When radical resection cannot be done at first diagnosis, the prognosis of patients with advanced gastric cancer (AGC) is rather poor [6].

Neoadjuvant chemotherapy (NAC) is a promising strategy of multimodality therapies. It is currently accepted as an effective treatment for ovarian, head and neck cancer 
and extremity tumors, considered to have many clinical advantages [7]. For potentially resectable patients, exposure to chemotherapy at the earliest time may prevent rapid growth of metastases after therapy of the primary sites and may also prevent the emergence of chemoresistant clones. Moreover, several studies have indicated that initially unresectable AGC can be successfully converted to resectable AGC by NAC and then treated with curative surgery [8-11]. However, the NAC regimens were various in these trials. S-1 plus cisplatin has been reported with $63 \%$ response rate (RR) and $31.0 \%$ 3-year overall survival (OS) rate in initially unresectable AGC in a phase II clinical trial [12]. Further studies indicated that the addition of docetaxel to cisplatin and S-1 could improve the outcome of patients with unresectable gastric cancer $[13,14]$. In contrast [15], the addition of epirubicin to XELOX (capecitabine and oxaliplatin) didn't improve effects in RR, radical resection rate, and OS benefits. And the chemotherapy-related toxicity caused by this tripledrug regimen was higher. So, the clinical efficacy of these chemotherapy regimens and whether multi-drug regimens bring more benefits than double-drug therapies do for these patients remain unknown.

So, we intended to find an easy and effective approach which can permit the use of a multi-drug regimen offering more effectiveness and decrease the chemotherapy-related toxicity simultaneously. The research conducted by Nakajima et al. gave a clue, in which the intra-arterial approach was used to inject etoposide and cisplatin for the treatment of AGC patients with excellent efficacy and reduced toxicity [16]. Thus, based on the same theory, we developed an NAC regimen (FLEEOX: 5-FU, leucovorin, etoposide, oxaliplatin, and epirubicin) via intra-arterial and intravenous administration for patients with initially unresectable
AGC. In our previous research, it has been demonstrated that FLEEOX could produce a favorable tumor RR with relatively mild toxicity profile for AGC patients with para-aortic lymph nodal metastasis [17]. In addition, fluorouracil plus oxaliplatin is a recommended two-drug regimen for AGC patients according to the National Comprehensive Cancer Network (NCCN) Guideline Edition 2007, and the fluorouracil can be replaced with capecitabine. Therefore, the aim of this study was to evaluate the clinical efficacy of FLEEOX for patients with initially unresectable AGC compared with XELOX (capecitabine and oxaliplatin) by means of a case-matched study with propensity score matching (PSM). Besides, the chemotherapy-related toxicity was also analyzed.

\section{RESULTS}

\section{Patients}

From January 2004 to December 2012, a total of 523 patients receiving NAC for AGC in our institution were reviewed (Figure 1). Of them, 87 patients were excluded because of receiving other anti-tumor treatments, peritoneal dissemination, distance organs metastasis or para-aortic lymph nodal metastases (PLNM). The remaining 436 patients who underwent either FLEEOX $(n=100)$ or XELOX $(n=336)$ were eligible for inclusion in this research.

\section{Baseline characteristics}

The baseline characteristics before and after PSM were listed in Table 1. Before the matching, there were significant differences in gender, tumor location,

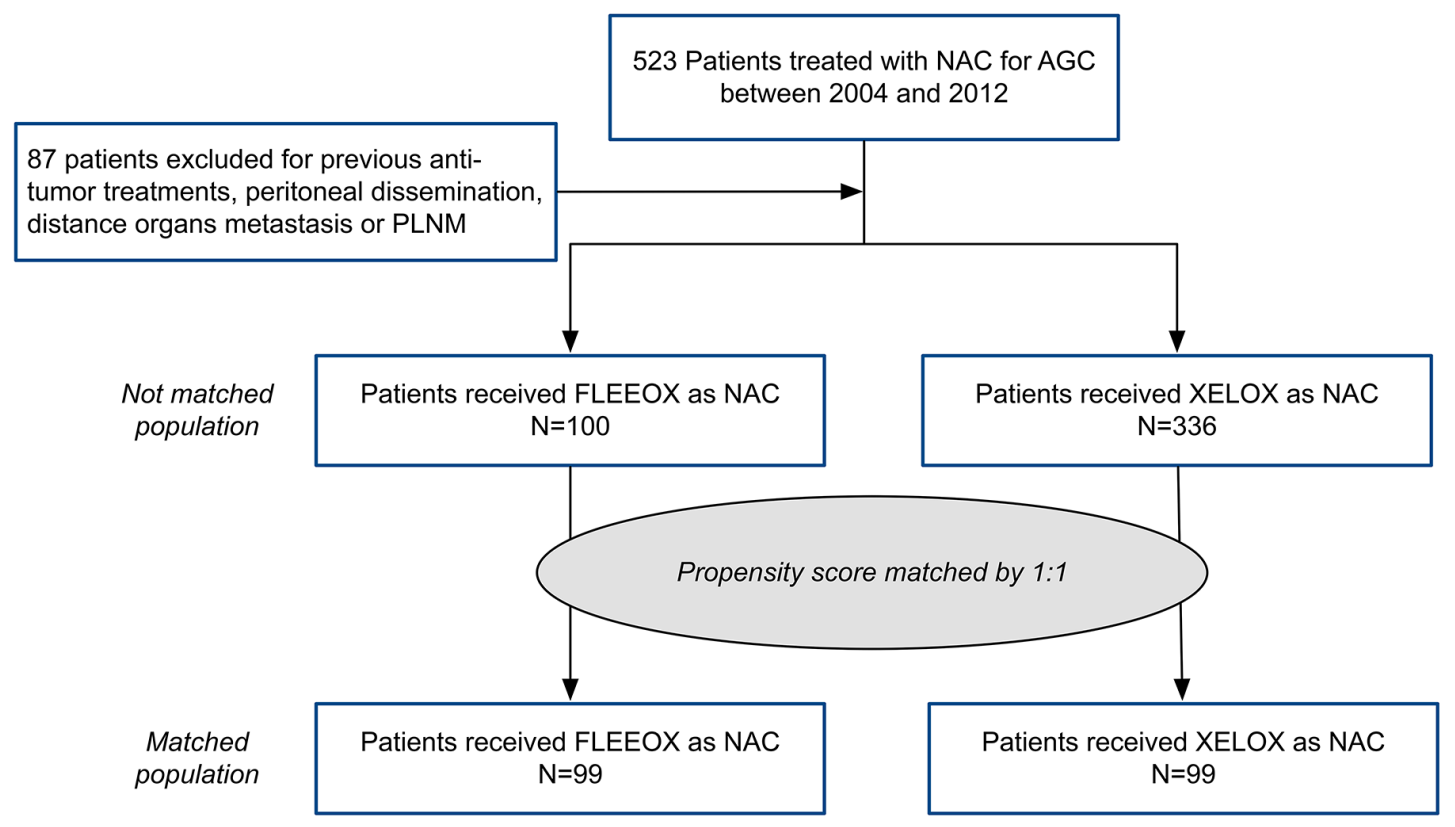

Figure 1: Patient study group CONSORT diagram. 
Table 1: The characteristics of the patients before and after PSM

\begin{tabular}{|c|c|c|c|c|c|c|}
\hline \multirow[t]{2}{*}{ Variable } & \multicolumn{3}{|c|}{ Before PSM } & \multicolumn{3}{|c|}{ After PSM } \\
\hline & $\begin{array}{c}\text { FLEEOX } \\
(n=100)(\%)\end{array}$ & $\begin{array}{c}\text { XELOX } \\
(n=336)(\%)\end{array}$ & $P$ & $\begin{array}{c}\text { FLEEOX } \\
(n=99)(\%)\end{array}$ & $\begin{array}{c}\text { XELOX }(n=99) \\
(\%)\end{array}$ & $P$ \\
\hline $\begin{array}{l}\text { Age } \\
\text { (mean years } \pm \text { SD) }\end{array}$ & $60.89 \pm 10.37$ & $59.99 \pm 9.19$ & 0.357 & $60.98 \pm 10.43$ & $59.74 \pm 9.54$ & 0.383 \\
\hline Gender & & & $<0.001$ & & & 0.126 \\
\hline Male & $70(70.0)$ & $294(87.5)$ & & $70(70.7)$ & $78(78.8)$ & \\
\hline Female & $30(30.0)$ & $42(12.5)$ & & $29(29.3)$ & $21(21.2)$ & \\
\hline Tumor location & & & $<0.001$ & & & 0.583 \\
\hline Upper third & $20(20.0)$ & $114(32.1)$ & & $26(26.3)$ & $23(23.2)$ & \\
\hline Middle third & $54(54.0)$ & $181(53.9)$ & & $54(54.5)$ & $61(61.6)$ & \\
\hline Lower third & $26(26.0)$ & $61(14.0)$ & & $19(19.2)$ & $15(15.2)$ & \\
\hline Differentiation & & & $<0.001$ & & & 0.151 \\
\hline High-medium & $26(26.0)$ & $111(33.0)$ & & $26(26.3)$ & $23(23.2)$ & \\
\hline Low & $73(73.0)$ & $160(47.6)$ & & $72(72.7)$ & $70(70.7)$ & \\
\hline Undifferentiating & $1(1.0)$ & $65(19.3)$ & & $1(1.0)$ & $6(6.1)$ & \\
\hline Clinical T classification & & & 0.450 & & & 0.387 \\
\hline cT3 & $40(40.0)$ & $139(41.4)$ & & $40(40.4)$ & $43(43.4)$ & \\
\hline cT4 & $60(60.0)$ & $197(58.6)$ & & $59(59.6)$ & $56(56.6)$ & \\
\hline Clinical $\mathrm{N}$ classification & & & 0.369 & & & 0.257 \\
\hline $\mathrm{cN} 1$ & $24(24.0)$ & $72(21.4)$ & & $24(24.2)$ & $22(22.2)$ & \\
\hline $\mathrm{cN} 2$ & $39(39.0)$ & $113(33.6)$ & & $38(38.4)$ & $29(29.3)$ & \\
\hline $\mathrm{cN} 3$ & $37(37.0)$ & $151(44.9)$ & & $37(37.4)$ & $48(48.5)$ & \\
\hline Cause of unresection & & & $<0.001$ & & & 0.173 \\
\hline Local advance & $15(15.0)$ & $132(39.3)$ & & $15(15.2)$ & $22(22.2)$ & \\
\hline Bulky lymph nodes & $44(44.0)$ & $117(34.8)$ & & $44(44.4)$ & $32(32.3)$ & \\
\hline Both & $41(41.0)$ & $87(25.9)$ & & $40(40.4)$ & $45(45.5)$ & \\
\hline
\end{tabular}

PSM, propensity score matching; FLEEOX, 5-Fu, leucovorin, etoposide, epirubicin and oxaliplatin; XELOX, capecitabine and oxaliplatin

differentiation, and cause of unresection between FLEEOX and XELOX groups $(P<0.001)$, whereas the clinical $\mathrm{T}$ and $\mathrm{N}$ classification were similar. The disparities were resolved, however, after the PSM performed, and no differences $(P>0.05)$ in the baseline characteristics were observed between the two matched groups.

\section{Tumor response to NAC}

The results showed advantageous tumor response to NAC in FLEEOX group $(P=0.026)$ (Table 2$) .13(13.1 \%)$ patients achieved complete response (CR) in FLEEOX group, which was far more than in XELOX group (3 cases, $3 \%$ ). The RR was $80.8 \%$ and the disease control rate (DCR) was $89.9 \%$ in FLEEOX group, whereas in XELOX group, the RR and the DCR were $68.7 \%$ and $84.8 \%$, respectively. Therefore, the results suggested a higher RR $(P=0.018)$ in FLEEOX group.

\section{Surgical characteristics and pathological response}

Finally, 80 cases in FLEEOX group and 63 in XELOX group received radical resection, and the radical resection rate in the front group was dramatically higher $(80.8 \%$ vs. $63.6 \%, P<0.001)$ (Table 3$)$. R0 surgical rate was also spotted higher in FLEEOX group (69/80, 86.3\% vs. $38 / 63,60.3 \%, P<0.001)$. Meanwhile, more grade 3 pathological response occurred in FLEEOX group (11/80, 
Table 2: Tumor response to NAC in the two groups after PSM

\begin{tabular}{lccc}
\hline Variable & FLEEOX $(\mathbf{n = 9 9 )}(\mathbf{\%})$ & XELOX $(\mathbf{n = 9 9 )}(\mathbf{\%})$ & $\boldsymbol{P}$ \\
\hline Response to NAC & & $\mathbf{0 . 0 2 6}$ \\
CR & $13(13.1)$ & $3(3.0)$ & $65(65.7)$ \\
PR & $67(67.7)$ & $16(16.2)$ & $15(15.2)$ \\
SD & $9(9.1)$ & $68(68.7)$ & $\mathbf{0 . 0 1 8}$ \\
PD & $10(10.1)$ & $84(84.8)$ & 0.196 \\
RR (CR plus PR) & $80(80.8)$ & \\
DCR (CR plus PR plus SD) & $89(89.9)$ &
\end{tabular}

PSM, propensity score matching; FLEEOX, 5-Fu, leucovorin, etoposide, epirubicin and oxaliplatin; XELOX, capecitabine and oxaliplatin; CR, complete response; PR, partial response; SD, stable disease; PD, progression disease; RR, response rate; $\mathrm{DCR}$, disease control rate

$13.8 \%$ vs. $3 / 63,4.8 \%, P<0.001)$. The mean number of dissected lymph nodes in FLEEOX group was less than XELOX group $(14.37 \pm 6.24$ vs. $20.55 \pm 10.96, P<0.001)$, whereas the mean number of positive lymph nodes was closed in the both groups (4.23 \pm 4.52 vs. $4.89 \pm 6.47$, $\mathrm{P}=0.473)$. The rates of pathological $\mathrm{T}$ and $\mathrm{N}$ classification were similar between the two groups $(P=0.409$ and 0.061 , respectively).

\section{Chemotherapy-related toxicity}

Overall, no chemotherapy-related deaths and no dropping out of treatment due to adverse events were spotted (Table 4). But, patients in FLEEOX group experienced higher incidence of leukocytopenia (17.2\% vs. $7.1 \%, P=0.024)$, nausea $(17.2 \%$ vs. $6.1 \%, P=0.012)$ and vomiting $(22.2 \%$ vs. $10.1 \%, P=0.016)$. The overall chemotherapy-related toxicity was dramatically higher in FLEEOX group than XELOX group (71.7\% vs. $35.4 \%$, $P<0.001)$.

\section{Overall survival (OS)}

After a median follow-up of 18 months (range 3.285 months), 150 patients (69 in FLEEOX group and 81 in XELOX group) passed away. The median OS time was 30.0 months (95\% CI 25.5-34.5) in FLEEOX group and 25.1 months (95\% CI 19.3-30.8) in XELOX group $(P<0.001$, Figure 2). Subgroup analysis showed that the patients who received radical surgery had a dramatically longer median OS time than other patients (Radical surgery: 31.3 months vs. Palliative surgery: 18.5 months vs. Non-surgery: 10 months, $P<0.001$, Figure 3 ). Tumor response had an essential influence on the OS time. Patients who achieved CR or PR had a longer median OS time than patients who achieved SD or PD (31.5 months vs. 20 months, $P<0.001$, Figure 4).

\section{DISCUSSION}

The optimal regimen of NAC for patients with initially unresectable AGC is still a matter of debate. The present study indicated that FLEEOX showed more advantages in terms of $\mathrm{RR}$, radical resection rate, $\mathrm{R} 0$ resection rate and $\mathrm{OS}$ as compared with XELOX. But a higher overall chemotherapyrelated toxicity was also observed in FLEEOX, although none of the patients dropped out of the treatment because of chemotherapy-related toxicity.

In many clinical trials, the RR to NAC for local AGC patients remains around 60\%[18-20]. In the study conducted by Nashimoto A et al., S-1 combined cisplatin to treat local AGC patients offered $62.5 \%$ of RR [18]. In addition, a better partial RR $(77.8 \%)$ has been reported in a phase I clinical trial [21], which evaluated the efficacy of FOLFOX4 (oxaliplatin/leucovorin/fluorouracil) for locally unresectable AGC patients. But the sample size in this study was very small $(n=9)$. Our results indicated that RR was $80.8 \%$ in FLEEOX group, whereas the $\mathrm{RR}$ in XELOX group was $63.6 \%$ which was similar to other studies. The high RR in FLEEOX group might be attributed to several reasons. First, the five-drug combination regimen used in this study may be more efficient than two- or three-drug therapies. The mixture of chemotherapeutic agents with different drug mechanisms may offer a combined effect to influence various synthesis procedures in tumor cells and improve the tumor response to NAC. For example, oxaliplatin and epirubicin selectively inhibit the synthesis of deoxyribonucleic acid (DNA) and their cytotoxicity is cell-cycle nonspecific. At high concentration of these drugs, cellular RNA and protein syntheses are also suppressed. Furthermore, etoposide inhibits DNA topoisomerase II, thereby inhibiting DNA re-ligation and affecting mainly the S and G2 phases of tumor cells. Second, the chemotherapy approach via intra-arterial injection increases the regional 
Table 3: The characteristics of surgery and pathological response to NAC for the patients received radical surgery after PSM

\begin{tabular}{|c|c|c|c|}
\hline Variable & FLEEOX $(\mathrm{n}=80)(\%)$ & $\operatorname{XELOX}(n=63)(\%)$ & $P$ \\
\hline \multicolumn{4}{|l|}{ Patients received surgery } \\
\hline Radical surgery & $80(80.8)$ & $63(63.6)$ & $<0.001$ \\
\hline Palliative surgery & $8(8.1)$ & $16(16.2)$ & $<0.001$ \\
\hline No surgery & $11(11.1)$ & $20(20.2)$ & \\
\hline \multicolumn{4}{|l|}{ Pathological response } \\
\hline Grade 3 & $11(13.8)$ & $3(4.8)$ & $<0.001$ \\
\hline Grade 2 & $45(56.2)$ & $38(60.3)$ & \\
\hline Grade 1 & $24(30.0)$ & $22(34.9)$ & \\
\hline \multicolumn{4}{|l|}{ Residual tumor classification } \\
\hline R0 & $69(69.7)$ & $38(38.4)$ & $<0.001$ \\
\hline R1 & $7(7.07)$ & $12(12.1)$ & \\
\hline R2 & $4(4.04)$ & $13(13.1)$ & \\
\hline TDLNs (mean \pm SD) & $14.37 \pm 6.24$ & $20.55 \pm 10.96$ & $<0.001$ \\
\hline Positive LNs (mean \pm SD) & $4.23 \pm 4.52$ & $4.89 \pm 6.47$ & 0.473 \\
\hline Pathological T classification & & & 0.409 \\
\hline урТ0 & $11(13.8)$ & $4(6.3)$ & \\
\hline ypT1 & $15(18.8)$ & $7(11.1)$ & \\
\hline урT2 & $14(17.5)$ & $15(23.8)$ & \\
\hline урT3 & $16(20.0)$ & $18(28.6)$ & \\
\hline урT4a & $21(26.3)$ & $16(25.4)$ & \\
\hline урT4b & $3(3.8)$ & $3(4.8)$ & \\
\hline Pathological N classification & & & 0.061 \\
\hline ypN0 & $20(25.0)$ & $18(28.6)$ & \\
\hline ypN1 & $20(25.0)$ & $19(30.2)$ & \\
\hline ypN2 & $23(28.8)$ & $9(14.3)$ & \\
\hline ypN3a & $8(10.0)$ & $14(22.2)$ & \\
\hline ypN3b & $9(11.2)$ & $3(4.8)$ & \\
\hline
\end{tabular}

PSM, propensity score matching; FLEEOX, 5-Fu, leucovorin, etoposide, epirubicin and oxaliplatin; XELOX, capecitabine and oxaliplatin; TDLNs, total dissected lymph nodes; LNs, lymph nodes

pharmaceutical concentration and strengthens local effects on the primary tumor and regional metastatic lymph nodes. A pilot study in Japan [22], which based on the same theory as our protocol that the aggressive regional treatment may improve the therapeutic efficacy, showed a high RR of $80 \%$ by the addition of local radiotherapy to oral administration of S-1 for gastric cancer patients with severe local infiltration and metastasis. Third, the hepatic first-pass effect of chemotherapeutic drugs, which metabolizes most of the agents, can be partially avoided via intra-arterial administration.
A high RR usually brings satisfactory radical resection rate and $\mathrm{R} 0$ resection rate. In the present study, we found that radical resection and R0 rates were $80.8 \%$ and $69.7 \%$ in FLEEOX group, respectively, which are higher than other studies. A phase II study [12] indicated that twenty-seven initially unresectable AGC patients treated with S-1 plus cisplatin had $63.0 \%$ response rate and $48 \% \mathrm{R} 0$ rate, and another research [10] showed that R0 rate was 63\%, in which DXP (docetaxel, cisplatin, and capecitabine) was used as NAC for forty-nine such cases. We also determined that patients could have a 
Table 4: Grade $3 / 4$ adverse events in the patients after PSM

\begin{tabular}{lccc}
\hline Variable & FLEEOX $(\mathbf{n}=\mathbf{9 9}) \mathbf{( \% )}$ & XELOX $(\mathbf{n = 9 9 )}(\mathbf{\%})$ & $\boldsymbol{P}$ \\
\hline Overall toxicity & $71(71.7)$ & $35(35.4)$ & $<\mathbf{0 . 0 0 1}$ \\
Related deaths & $0(0 \%)$ & $0(0 \%)$ & NA \\
Aborting of NAC & $0(0 \%)$ & $0(0 \%)$ & NA \\
Hematological & & & \\
$\quad$ Leukocytopenia & $17(17.2)$ & $7(7.1)$ & $\mathbf{0 . 0 2 4}$ \\
Thrombocytopenia & $9(9.1)$ & $7(7.1)$ & 0.398 \\
Non-hematological & & & \\
Nausea & $17(17.2)$ & $6(6.1)$ & $\mathbf{0 . 0 1 2}$ \\
Vomiting & $22(22.2)$ & $10(10.1)$ & $\mathbf{0 . 0 1 6}$ \\
Diarrhea & $4(4.0)$ & $4(4.0)$ & 0.640 \\
Hepatic inadequacy & $2(2.0)$ & $1(1.0)$ & 0.500 \\
Renal dysfunction & $0(0.0)$ & $0(0.0)$ & - \\
\hline
\end{tabular}

PSM, propensity score matching; FLEEOX, 5-Fu, leucovorin, etoposide, epirubicin and oxaliplatin; XELOX, capecitabine and oxaliplatin; NA, no available; NAC, neoadjuvant chemotherapy

survival benefit from radical resection after response to the NAC. Median OS time could be prolonged from 10 to 31.5 months when conversion chemotherapy and radical resection were accomplished, and this result was similar to the previous study [12]. Patients in FLEEOX group had longer median OS time than XELOX group (30 vs. 25.1 months, $P<0.001$ ) because of a higher radical resection rate and better pathological response, which indicated that the front regimen had more efficacy in patients with initially unresectable AGC. Meanwhile, the radiological response could predict that patients who obtained $\mathrm{CR}$ or PR would have better OS than these achieved stable disease (SD) or progressive disease (PD) (31.5 vs. 20 months, $P<0.001)$. This means that the response to NAC may predict survival before curative resection of gastric cancer.

Our results also indicated that fewer lymph nodes were harvested in FLEEOX group than that in XELOX group. The possible reason is that more bulky lymph nodes and/or merging of metastatic lymph nodes occurred in FLEEOX group (Table 1). For example, metastatic No. 7, No.8 and No.9 station lymph nodes often merge and encompass the celiac artery as one reason for unresectable AGC. Even after NAC, this combination of metastatic

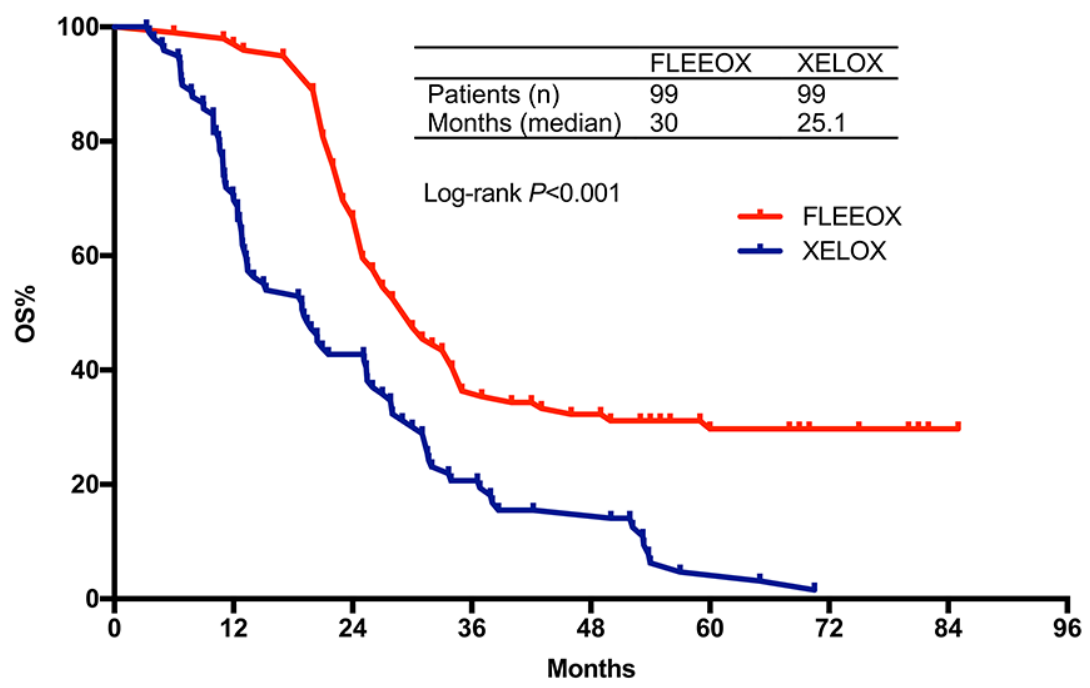

Figure 2: Overall survival according to treatment. 
lymph nodes usually cannot be divided and will be considered and reported as a signal metastatic lymph node by pathologists during pathological evaluation. So, the count of harvested lymph nodes in FLEEOX group was less than the actual number when statistical analysis was performed, although the same D2 radical resection was used in the both groups.

In addition, it was apparently known that more aggressive therapy containing multi-drugs caused more adverse events. Our results indicated that NAC-related toxicity, especially in leukocytopenia, nausea and vomiting, tended to be more frequent in FLEEOX group than XELOX group as well the results from other studies
[23]. The high frequency of overall chemotherapy-related toxicity in FLEEOX group was ascribed to the high regional concentration of drugs and local irritant effect. But during the period of study, none of the patients quit the treatment for intolerable chemotherapy-related toxicity. And most of the toxicity including leukocytopenia, nausea and vomiting could be alleviated by granulocyte stimulating factor or antiemetic drugs. In another hand, the intra-arterial approach also helped reduce the drug dosages without affecting the therapeutic efficacy. And this could partially explain why the systemic adverse reaction was not increased in FLEEOX regimen, thrombocytopenia, hepatic inadequacy and renal dysfunction, for instance.

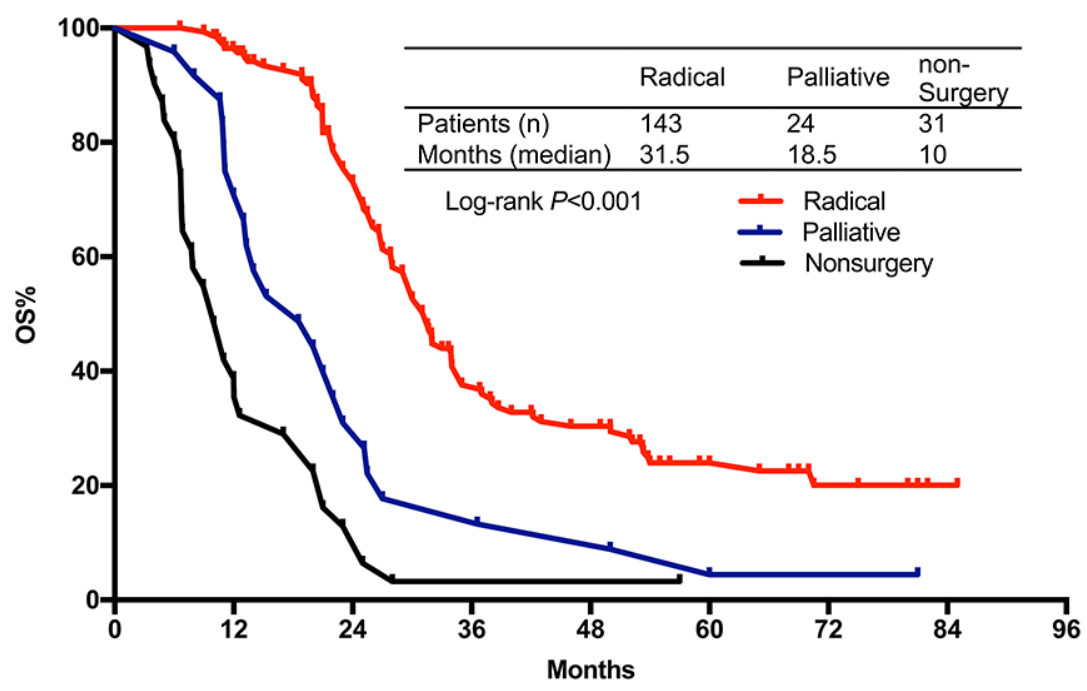

Figure 3: Overall survival stratified by CR+PR vs. SD+PD.

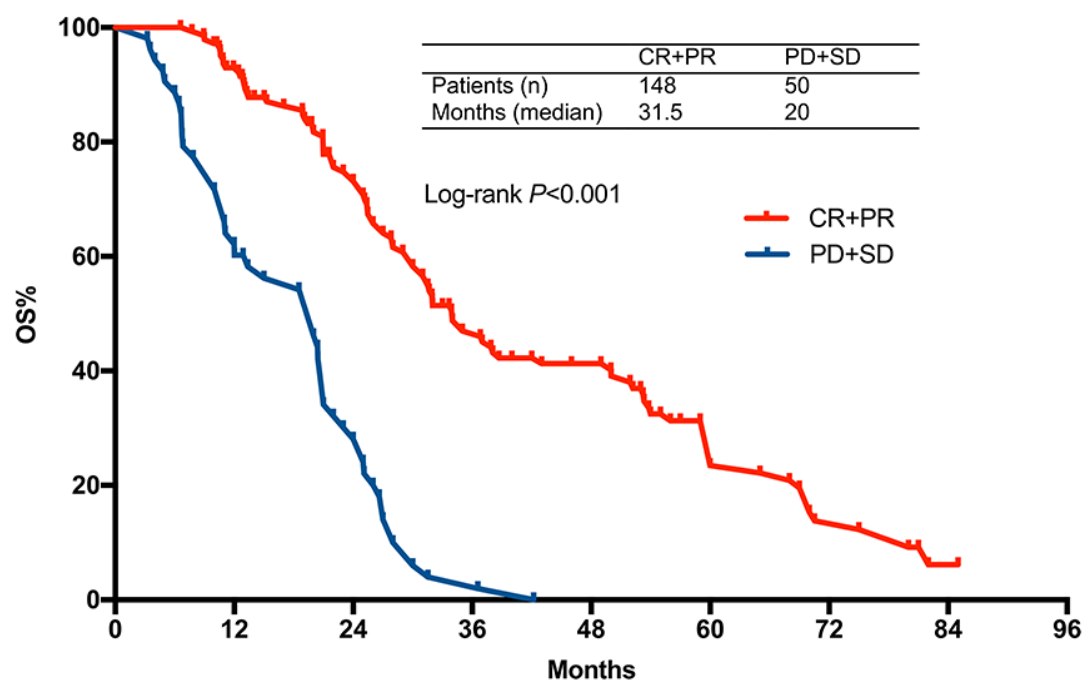

Figure 4: Overall survival stratified by radical resection vs. palliative surgery vs. non-surgery. 
Since it is a retrospective study, there are some limitations that cannot be avoided. First, non-randomized and unblinded setting may have a great chance producing selection bias. But, PSM used in this study may well balance the basic characteristics between the two groups. Second, PSM is usually used in large sample studies to control for pre-group differences. In smaller sample size studies, such attrition leaves too few cases for meaningful analysis. But in our study, there were 99 cases in each group after matching which means few subjects missing in the experimental group. Furthermore, Pirracchio et al. indicated that even in the case of small study samples or low prevalence of treatment, PSM can yield correct estimations of treatment effect unless the true confounders and the variables related only to the outcome are not included in the PS model. And no substantial increasing in the Type I error rate was observed as the sample size decreased from 1,000 to 40 subjects.

In conclusion, our research suggested that initially unresectable AGC patients can obtain more benefits from FLEEOX regimen. The more aggressive treatment including five chemotherapeutic drugs would improve $\mathrm{RR}$, radical resection rate, $\mathrm{R} 0$ resection rate and $\mathrm{OS}$. In addition, FLEEOX produces more toxicity effects when compared to XELOX, although no chemotherapy-related deaths and aborting of NAC were observed. Further randomized clinical trials about FLEEOX regimen are necessary.

\section{PATIENTS AND METHODS}

\section{Patients and pretreatment evaluation}

This retrospective study was conducted at a single institution. From January 2004 to December 2012, patients with initially unresectable AGC who received NAC were reviewed from our database. As opposed to earlystage gastric cancer, AGC encompasses locally advanced and/or lymph node metastasis. And based on TNM Classification of malignant tumors $7^{\text {th }}\left(\mathrm{TNM} 7^{\text {th }}\right)$ [24], AGC in this study includes T3-4, N1-3 and M0. All patients were enrolled according to the following eligibility criteria: 1) histologically proven gastric cancer, 2) evaluated as initially unresectable AGC by abdominal enhanced computed tomography (CT) scan, 3) have signed informed consent before the beginning of treatment, 4) no prior anti-tumor therapy, 5) age 20-70 years old, 6) Karnofsky performance status $(\mathrm{KPS})>70,7)$ no serious concomitant diseases that make survival period less than 5 years.

Patients are considered as initially unresectable AGC when one or more of the following criteria exist: 1) primary cancer directly infiltrates any one or more of the adjacent structures such as pancreatic head, hepatoduodenal ligament, abdominal main artery, and proximal segment of splenic artery, 2) bulky lymph nodes (larger than $3 \mathrm{~cm}$ ) or merging of metastatic lymph nodes encompassing vital blood vessels: celiac artery, hepatic artery, splenic artery root, hepatoduodenal ligament, and near portal segment of splenic vein.

Exclusion criteria included: 1) the PLNM by CT scan or left supraclavicular lymph nodal metastasis by biopsy, 2) peritoneal dissemination, 3) other organs metastasis, such as liver and lung, 4) serious or uncontrolled systemic diseases, 5) chemotherapy drug allergies, 6) pregnant or lactating, and 7) with other malignancies contemporaneously, 8) receiving other regimens neither FLEEOX or XELOX. The clinical stage was determined by the TNM Classification of malignant tumors $7^{\text {th }}\left(\right.$ TNM $\left.7^{\text {th }}\right)[24]$.

\section{Propensity score matching}

The propensity score, as defined by Rosenbaum and Rubin [25], is the individual probability of receiving the treatment of interest conditional on the observed baseline covariates. It has been demonstrated that, within the strata of subjects matched on the propensity score, distributions of these covariates tend to be similar between treated and untreated. In short, PSM was developed to investigate causal relationships between therapeutic protocols and outcomes in a retrospective study other than a randomized controlled trial $[26,27]$.

In this study, PSM was used to generate a matched pair of cases to compare the clinical efficacy between patients receiving FLEEOX and XELOX. Propensity scores were estimated based on age, gender, tumor location, differentiation, clinical $\mathrm{T}$ classification, clinical $\mathrm{N}$ classification, and cause of unresection. One-to-one matching without replacement was performed using a 0.05 caliper width [28]. And the score-matched pairs were used in subsequent analyses.

\section{Neoadjuvant chemotherapy}

FLEEOX: 5-Fu (370 mg/m²) and leucovorin (200 $\mathrm{mg} / \mathrm{m}^{2}$ ) were administered by intravenous infusion on day 1-5. Intra-arterial administration of etoposide $\left(80 \mathrm{mg} / \mathrm{m}^{2}\right)$, oxaliplatin $\left(80 \mathrm{mg} / \mathrm{m}^{2}\right)$, and epirubicin $\left(30 \mathrm{mg} / \mathrm{m}^{2}\right)$ was performed by Seldinger method on day 6 and 20, the catheter was inserted through femoral artery into the celiac artery and the chemicals were injected initially at relatively high doses, followed by 14 days' rest.

The blood vessel to inject the drugs was selected by experienced radiologists according to the angiographic results. Usually, chemicals were injected into the left gastric artery for cancer of the upper and middle stomach, while for cancer at the lower stomach the right gastroepiploic artery was selected. Drugs were injected in the order of oxaliplatin, etoposide, and epirubicin, and each injection took $5 \mathrm{~min}$.

XELOX: Intravenous administration of oxaliplatin $\left(130 \mathrm{mg} / \mathrm{m}^{2}\right)$ was performed on day 1 and capecitabine was taken orally $\left(1000 \mathrm{mg} / \mathrm{m}^{2}\right)$ twice a day on day 1 to 14 of a 21-day cycle. 


\section{Evaluation of tumor response and chemotherapy-related toxicity}

After two cycles of NAC, the tumor response to chemotherapy was evaluated using an abdominal and pelvic enhanced CT scan by two experienced radiologists, according to the Response Evaluation Criteria in Solid Tumors (RECIST) guideline 1.1 [29]. CR was defined as clinical complete regression of the disease. More than 30\% reduction in maximum transverse diameter of the primary tumor was defined as PR, while more than $20 \%$ increase in maximum transverse diameter of the primary tumor or the appearance of new lesions was considered as PD. Other cases were included into SD. Radical resection (R0) was determined the operation leaving no macroscopical or microscopical tumor behind. All the chemotherapy-related adverse events during NAC were recorded daily and were evaluated according to National Cancer Institute Common Toxicity Criteria (NCI-CTC) version 3.0 [30].

\section{Surgical treatment and pathological response}

Resectability was assessed by a multidisciplinary team (MDT) after two courses of NAC. Cases with unresectable tumors continued NAC with resectability evaluation every two cycles for a maximum duration of six cycles or until progression. The patients identified as resectable by MDT would receive open surgery. The final surgical procedure would be determined by surgeons during surgery. Patients who were found with unresectable encompassing of vital organs and/or blood vessels during surgery would receive palliative treatment, while others would be given an extended lymph node resection (D2) plus gastrectomy. Surgical specimens were assessed histologically, and the pathological response was evaluated according to the histological criteria of JCGC (Japanese classification of gastric carcinoma, 3rd English edition) [31]. Grade 0, no effect; grade 1, slight effect (grade 1a, viable tumor cells occupy more than $2 / 3$ of the entire cancer area; grade $1 \mathrm{~b}$, viable tumor cells remain in more than $1 / 3$ but less than $2 / 3$ of the entire cancer area); grade 2 , considerable effect (viable tumor cells remain in less than $1 / 3$ of the entire cancer area); and grade 3 , complete response (no viable tumor cells remain).

\section{Postoperative treatment and follow-up}

After surgical treatment, patients were given six cycles of XELOX as adjuvant chemotherapy no matter what the NAC regimen was. Patients who could not undergo a radical resection received other chemotherapy regimens and/or best supportive care. All patients were followed up regularly. Tumor markers including serum carcinoembryonic antigen (CEA) and CA199 were examined every 3 months. Chest X-ray and abdominal/pelvic were examined every 6 months. Upper gastrointestinal endoscopy was conducted each year.

\section{Statistical analysis}

All analyses were performed by using SPSS version 24.0 for MAC (SPSS Inc., Chicago, IL, USA) and GraphPad Prism version 7.0 for MAC (GraphPad Software Inc., San Diego, CA, USA). The chi-squared test and Student's t-test were used for comparisons between the two groups. PSM was applied with a caliper 0.05 . Kaplan-Meier analysis with log-rank testing was used for OS. Continuous variables are expressed as mean \pm standard deviation. $P$ value $<0.05$ was considered statistically significant.

\section{Abbreviations}

FLEEOX, 5-Fu, leucovorin, etoposide, oxaliplatin, and epirubicin; XELOX, capecitabine and oxaliplatin; NAC, neoadjuvant chemotherapy; AGC, advanced gastric cancer; OS, overall survival; ECF, epirubicin, cisplatin and continuous 5-fluorouracil; EAP, etoposide, doxorubicin, and cisplatin; PSM, propensity score matching; PLNM, para-aortic lymph nodal metastases; $\mathrm{RR}$, response rate; $\mathrm{CR}$, complete response; $\mathrm{PR}$, partial response; $\mathrm{PD}$, progressive disease; $\mathrm{SD}$, stable disease; DNA, deoxyribonucleic acid.

\section{Authors' contributions}

Yang Li, Jun Chen, Chaogang Fan and Guoli Li contributed to the design and execution of the study. Qi $\mathrm{He}$ and Xiang Ji accrued patients, collected clinical data and specimens. Qi He, Xiang Ji and Xulin Wang carried out the experiments. Yang Li and Jun Chen analyzed the data and drafted the manuscript. Guoli Li and Chaogang Fan coordinated and oversaw the execution of the study. All authors read and approved the final manuscript.

\section{ACKNOWLEDGMENTS}

The protocol was approved by the Chinese Ethics Committee of Registering Clinical Trials, ChiECRCT, and Independent Institutional Review Board (IRB) of Jinling Hospital. This trial is registered on clinicalTrialecrforg and has the identification number ChiCTR-TRC-12002046.

\section{CONFLICTS OF INTEREST}

The authors made no disclosures.

\section{FUNDING SUPPORT}

No specific funding was disclosed.

\section{Informed consent}

Additional informed consent was obtained from all individual participants or their family members (if patients 
passed away) for whom identifying information is included in this article.

\section{REFERENCES}

1. Ferlay J, Soerjomataram I, Dikshit R, Eser S, Mathers C, Rebelo M, Parkin DM, Forman D, Bray F. Cancer incidence and mortality worldwide: sources, methods and major patterns in GLOBOCAN 2012. Int J Cancer. 2015; 136: E359-86. https://doi.org/10.1002/ijc.29210.

2. Chen W, Zheng R, Baade PD, Zhang S, Zeng H, Bray F, Jemal A, Yu XQ, He J. Cancer statistics in China, 2015. CA Cancer J Clin. 2016; 66: 115-32. https://doi.org/10.3322/ caac.21338.

3. Chen W, Zheng R, Zhang S, Zhao P, Li G, Wu L, He J. Report of incidence and mortality in China cancer registries, 2009. Chin J Cancer Res. 2013; 25: 10-21. https://doi. org/10.3978/j.issn.1000-9604.2012.12.04.

4. Zhang Q, Chen ZY, Chen CD, Liu T, Tang XW, Ren YT, Huang SL, Cui XB, An SL, Xiao B, Bai Y, Liu SD, Jiang B, et al. Training in early gastric cancer diagnosis improves the detection rate of early gastric cancer: an observational study in China. Medicine (Baltimore). 2015; 94: e384. https://doi. org/10.1097/MD.0000000000000384.

5. van de Velde CJ, Peeters KC. The gastric cancer treatment controversy. J Clin Oncol. 2003; 21: 2234-6. https://doi. org/10.1200/JCO.2003.91.138.

6. Isobe Y, Nashimoto A, Akazawa K, Oda I, Hayashi K, Miyashiro I, Katai H, Tsujitani S, Kodera Y, Seto Y, Kaminishi M. Gastric cancer treatment in Japan: 2008 annual report of the JGCA nationwide registry. Gastric Cancer. 2011; 14: 301-16. https://doi.org/10.1007/ s10120-011-0085-6.

7. Yamao T. (1999). Rationale for Neoadjuvant Chemotherapy for Advanced Gastric Cancer: Springer Japan.

8. Gallardo-Rincon D, Onate-Ocana LF, Calderillo-Ruiz G. Neoadjuvant chemotherapy with P-ELF (cisplatin, etoposide, leucovorin, 5-fluorouracil) followed by radical resection in patients with initially unresectable gastric adenocarcinoma: a phase II study. Ann Surg Oncol. 2000; 7: 45-50.

9. Ajani JA, Mayer RJ, Ota DM, Steele GD, Evans D, Roh M, Sugarbaker DJ, Dumas P, Gray C, Vena DA, et al. Preoperative and postoperative combination chemotherapy for potentially resectable gastric carcinoma. J Natl Cancer Inst. 1993; 85: 1839-44.

10. Sym SJ, Chang HM, Ryu MH, Lee JL, Kim TW, Yook JH, Oh ST, Kim BS, Kang YK. Neoadjuvant docetaxel, capecitabine and cisplatin (DXP) in patients with unresectable locally advanced or metastatic gastric cancer. Ann Surg Oncol. 2010; 17: 1024-32. https://doi. org/10.1245/s10434-009-0838-1.

11. Xiong BH, Cheng Y, Ma L, Zhang CQ. An updated metaanalysis of randomized controlled trial assessing the effect of neoadjuvant chemotherapy in advanced gastric cancer. Cancer Invest. 2014; 32: 272-84. https://doi.org/10.3109/0 7357907.2014 .911877$.

12. Inoue $\mathrm{K}$, Nakane $\mathrm{Y}$, Kogire M, Fujitani K, Kimura Y, Imamura H, Tamura S, Okano S, Kwon AH, Kurokawa Y, Shimokawa T, Takiuchi H, Tsujinaka T, et al. Phase II trial of preoperative S-1 plus cisplatin followed by surgery for initially unresectable locally advanced gastric cancer. Eur J Surg Oncol. 2012; 38: 143-9. https://doi.org/10.1016/j. ejso.2011.11.009.

13. Oyama K, Fushida S, Kinoshita J, Makino I, Nakamura K, Hayashi H, Nakagawara H, Tajima H, Fujita H, Takamura H, Ninomiya I, Kitagawa H, Tani T, et al. Efficacy of preoperative chemotherapy with docetaxel, cisplatin, and S-1 (DCS therapy) and curative resection for gastric cancer with pathologically positive para-aortic lymph nodes. J Surg Oncol. 2012; 105: 535-41. https://doi.org/10.1002/ jso. 22125 .

14. Hamakawa T, Kurokawa Y, Takiguchi S, Takahashi T, Yamasaki M, Miyata H, Nakajima K, Mori M, Dok Y. [Combination chemotherapy using docetaxel, cisplatin, and S-1 for far advanced gastric cancer]. [Article in Japanese]. Gan To Kagaku Ryoho. 2011; 38: 2342-4.

15. Wang Y, Zhuang RY, Yu YY, Yu S, Hou J, Ji Y, Sun YH, Shen KT, Shen ZB, Liu FL. Efficacy of preoperative chemotherapy regimens in patients with initially unresectable locally advanced gastric adenocarcinoma: capecitabine and oxaliplatin (XELOX) or with epirubicin (EOX). Oncotarget. 2016; 7: 76298-76307. https://doi. org/10.18632/oncotarget.11818.

16. Ishihara S, Nakajima T, Ohta K, Tsuchiya S, Okumura S, Mizuno S, Toyoda S, Nishi M, Ohashi Y. [Evaluation of effective neo-adjuvant chemotherapy (FLEP therapy) in the treatment of advanced gastric cancer]. [Article in Japanese]. Gan To Kagaku Ryoho. 1991; 18: 1748-52.

17. He Q, Ma L, Li Y, Li G. A pilot study of an individualized comprehensive treatment for advanced gastric cancer with para-aortic lymph node metastasis. BMC Gastroenterol. 2016; 16: 8. https://doi.org/10.1186/s12876-016-0422-7.

18. Nashimoto A, Yabusaki H, Nakagawa S, Takii Y, Tsuchiya Y, Otsuo T. Preoperative chemotherapy with S-1 and cisplatin for highly advanced gastric cancer. Anticancer Res. 2009; 29: 4689-96.

19. Chua YJ, Cunningham D. The UK NCRI MAGIC trial of perioperative chemotherapy in resectable gastric cancer: implications for clinical practice. Ann Surg Oncol. 2007; 14: 2687-90. https://doi.org/10.1245/s10434-007-9423-7.

20. Kang YK, Kang WK, Shin DB, Chen J, Xiong J, Wang J, Lichinitser M, Guan Z, Khasanov R, Zheng L, Philco-Salas M, Suarez T, Santamaria J, et al. Capecitabine/cisplatin versus 5-fluorouracil/cisplatin as first-line therapy in patients with advanced gastric cancer: a randomised phase III noninferiority trial. Ann Oncol. 2009; 20: 666-73. https:// doi.org/10.1093/annonc/mdn717. 
21. Chi Y, Yang J, Yang S, Sun Y, Jia B, Shi Y. Phase I dosefinding study of sorafenib with FOLFOX4 as first-line treatment in patients with unresectable locally advanced or metastatic gastric cancer. Chin J Cancer Res. 2015; 27: 23946. https://doi.org/10.3978/j.issn.1000-9604.2015.06.08.

22. Inoue $\mathrm{T}$, Yachida $\mathrm{S}$, Usuki $\mathrm{H}$, Kimura $\mathrm{T}$, Hagiike $\mathrm{M}$, Okano K, Suzuki Y. Pilot feasibility study of neoadjuvant chemoradiotherapy with S-1 in patients with locally advanced gastric cancer featuring adjacent tissue invasion or JGCA bulky N2 lymph node metastases. Ann Surg Oncol. 2012; 19: 2937-45. https://doi.org/10.1245/ s10434-012-2332-4.

23. Wang Y, Zhuang RY, Yu YY, Yu S, Hou J, Ji Y, Sun YH, Shen KT, Shen ZB, Liu FL, Zhao NQ, Liu TS. Efficacy of preoperative chemotherapy regimens in patients with initially unresectable locally advanced gastric adenocarcinoma: capecitabine and oxaliplatin (XELOX) or with epirubicin (EOX). Oncotarget. 2016; 7: 76298-307. https://doi.org/10.18632/oncotarget.11818.

24. Edge S, Byrd D, Compton C, Fritz A, Greene F, Trotti A. American Joint Committee on Cancer Staging Manual. 7th ed. New York: Springer. 2009.

25. Rosenbaum PR, Rubin DB. The central role of the propensity score in observational studies for causal effects. Biometrika. 1983; 70: 41-55.
26. Austin PC. A critical appraisal of propensity-score matching in the medical literature between 1996 and 2003. Stat Med. 2008; 27: 2037-49. https://doi.org/10.1002/sim.3150.

27. Baser $\mathrm{O}$. Too much ado about propensity score models? Comparing methods of propensity score matching. Value Health. 2006; 9: 377-85. https://doi. org/10.1111/j.1524-4733.2006.00130.x.

28. D'Agostino RB Jr. Propensity score methods for bias reduction in the comparison of a treatment to a nonrandomized control group. Stat Med. 1998; 17: 2265-81.

29. Eisenhauer EA, Therasse P, Bogaerts J, Schwartz LH, Sargent D, Ford R, Dancey J, Arbuck S, Gwyther S, Mooney M, Rubinstein L, Shankar L, Dodd L, et al. New response evaluation criteria in solid tumours: revised RECIST guideline (version 1.1). Eur J Cancer. 2009; 45: 228-47. https://doi.org/10.1016/j.ejca.2008.10.026.

30. Trotti A, Colevas AD, Setser A, Rusch V, Jaques D, Budach V, Langer C, Murphy B, Cumberlin R, Coleman CN, Rubin P. CTCAE v3.0: development of a comprehensive grading system for the adverse effects of cancer treatment. Semin Radiat Oncol. 2003; 13: 176-81. https://doi.org/10.1016/ S1053-4296(03)00031-6.

31. Japanese Gastric Cancer A. Japanese classification of gastric carcinoma: 3rd English edition. Gastric Cancer. 2011; 14: 101-12. https://doi.org/10.1007/s10120-011-0041-5. 